\section{Stellar beginnings}

André Maeder

The Physics of Stars. By A. C. Philips. Wiley: 1994. Pp. 208. £42.50, \$68.50 (hbk); £17.95, \$28.95 (pbk).

FAR-reaching consequences in stellar physics can be derived from first principles. The beautiful and straightforward connections between fundamental physics and the properties of stars are shown well in this book. It is pitched at undergraduates and successfully provides short accounts of selected topics. The author appreciates that the motivation to study physics is enhanced by astrophysical applications, while a proper understanding of how stars work in turn requires a clear and sound basis in physics.

After a good introductory chapter on some important astrophysical concepts, the author focuses on the properties of matter and radiation. He derives the various equations of state that have large consequences for stars, and provides a brief account of the main processes of heat transfer and the basic physics of nuclear processes, giving an overview of the main nuclear reactions during stellar evolution. On treating the applications to stellar structure, he emphasizes simple models and minimum and maximum stellar masses. He then makes the wise choice of jumping to the end-points of stellar evolution (white dwarfs, neutron stars and black holes), while showing how basic principles determine their main features.

Throughout there is a proper balance between text and equations. The sequence of arguments and the logical steps in the mathematics are developed with care so that they can be easily followed by a reader with a basic knowledge of physics. My only criticism is the lack of good, relevant figures.

Problems are posed at the end of each chapter, and hints at the answers are given at the back of the book. Many such books contain problems so difficult that nobody even tries to solve them; the ones here are interesting and easy enough to stimulate rather than discourage students.

André Maeder is at the Observatoire de Genève, $\mathrm{CH}$ des Maillettes 51, $\mathrm{CH}-1290$ Sauverny, Switzerland.

- Also just published: Astrophysical Jets edited by D Burgaretla, M. Livio and C. O'Dea. Proceedings of a meeting held in Baltimore in May 1992. CUP, £35, $\$ 59.95$; The Nature of Compact Objects in Active Galactic Nuclei edited by A. Robinson and R. J. Terlevich. Proceedings of the 23rd Herstmonceux conference, Cambridge, July 1992. CUP, £40, \$59.95; and Solar and Planetary Dynamos edited by M. R. E. Proctor, P. C. Matthews and A. M. Rucklidge Proceedings of a NATO meeting, Cambridge. September 1992. CUP, £35, \$49.95.

\title{
Big bodies, big brains
}

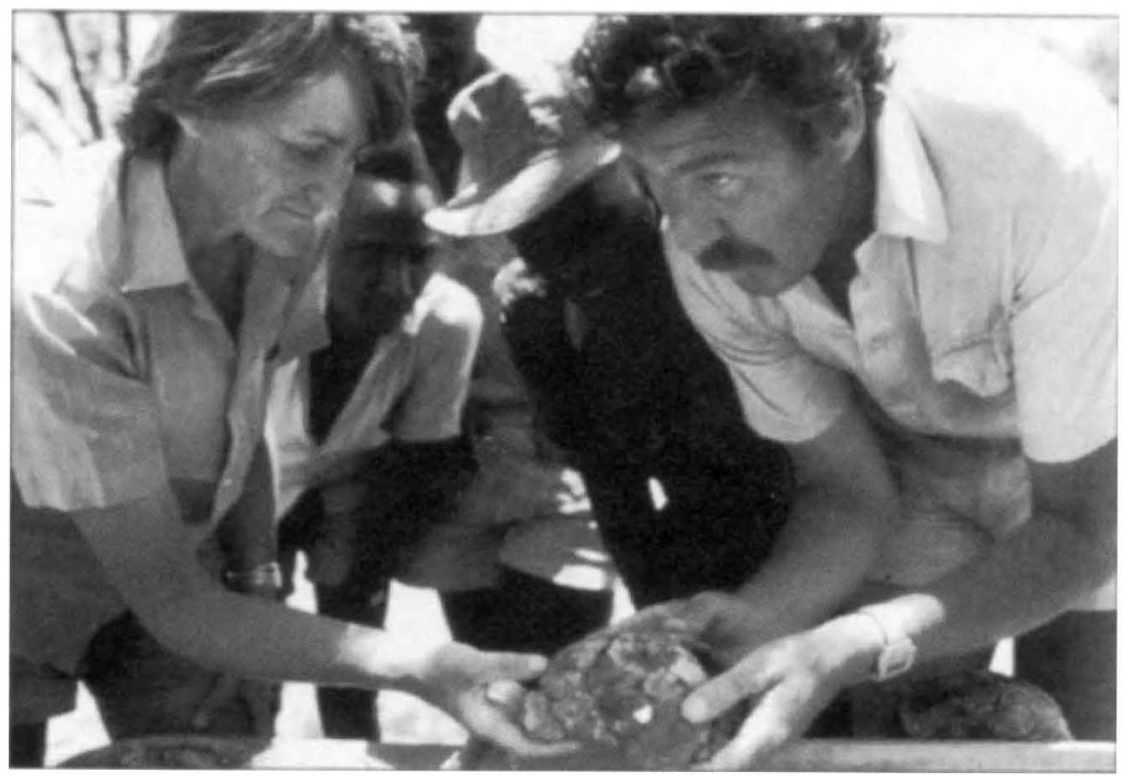

Meave Leakey and Alan Walker examining the cranium of the Homo erectus boy found during the 1984 field season at Nariokotome, Kenya. The skeleton itself is the subject of the book reviewed below, while this picture is taken from Ancestors: In Search of Human Origins written by the palaeoanthropologist Donald Johanson together with Lenora Johanson and Blake Edgar (Villard Books, \$27.50). Immaculately illustrated and produced, the volume was released to coincide with a recent three-part NOVA television series - hosted by Donald Johanson - on human evolution.

\section{Old boy}

\section{Bernard Wood}

The Nariokotome Homo Erectus

Skeleton. Edited by Alan Walker and Richard Leakey. Springer: 1993. Pp. 457. DM248.

EVOLUTIONARY history can often be retrieved by comparing closely related living forms. But modern humans differ substantially in both morphology and behaviour from their closest living relatives. the African apes, the chimpanzee and the gorilla. We are the sole living representative of our adaptive plateau, so details of our evolutionary history have to be sought from the palacontological record.

Fossil evidence usually comes in dribs and drabs, a tooth here and a fragment of limb bone there. The challenge to palaeontologists is to use these data to generate testable hypotheses about human evolution. There is always the possibility that limbs may be matched with the wrong body and teeth with the wrong head, thus creating combinations that did not exist in nature. Now and again. however, much better preserved fossils are recovered. The main value of a single skeleton is that it can confirm or refute links between cranial and postcranial remains as well as provide more reliable and otherwise unobtainable evi- dence about relationships between body shape and size.

The early hominid skeleton KNMWT15000 (referred to here as WT15K) is unrivalled among its contemporaries in its completeness. The first evidence of it, a small piece of cranial vault, was discovered by the veteran fossil-hunter Kamoya Kimeu in 1984 beside a tributary of the Nariokotome sand river on the west side of Lake Turkana in northern Kenya. Its recovery took five seasons of excavation and the removal, by hand, of 1.500 cubic metres of earth. Little of the feet, hands and forearms was recovered and there are no neck vertebrae, but, these deficiencies apart, the skeleton is remarkably complete. The geological reconstruction suggests that the skeleton was dismembered by decomposition and the components buried naturally in a swamp next to a grassy floodplain. Its geological age falls between 1.51 and 1.56 million years old. The cause of death is unknown but there is no evidence of violence: the only pathological sign is a small abscess cavity in the lower jaw.

Alan Walker and Richard Leakev, the editors of this volume, were both directly involved in unearthing and reconstructing the skeleton. The book is in two parts. the first describing the skeleton and its context and the second comprising the reports of eight analytical studies. It finishes with a summary by Alan Walker that puts the 